\title{
A!
}

This is an electronic reprint of the original article.

This reprint may differ from the original in pagination and typographic detail.

Sourander, M.; Vermasvuori, M.; Sauter, D.; Liikala, Teemu; Jämsä-Jounela, Sirkka-Liisa

\section{Fault Tolerant Control for a Dearomatisation Process}

Published in:

Journal of Process Control

Published: 01/01/2009

Document Version

Peer reviewed version

Please cite the original version:

Sourander, M., Vermasvuori, M., Sauter, D., Liikala, T., \& Jämsä-Jounela, S-L. (2009). Fault Tolerant Control for a Dearomatisation Process. Journal of Process Control, 19(7), 1091-1102.

This material is protected by copyright and other intellectual property rights, and duplication or sale of all or part of any of the repository collections is not permitted, except that material may be duplicated by you for your research use or educational purposes in electronic or print form. You must obtain permission for any other use. Electronic or print copies may not be offered, whether for sale or otherwise to anyone who is not an authorised user. 


\title{
Fault tolerant control for a dearomatisation process
}

\author{
M. Sourander ${ }^{\mathrm{c}}$, M. Vermasvuoria*, D. Sauter ${ }^{\mathrm{b}}$, T. Liikala ${ }^{\mathrm{c}}$, S.-L. Jämsä-Jounela ${ }^{\mathrm{a}}$ \\ a Aalto University, Department of Chemical Technology, Kemistintie 1, FIN-02150 Espoo, Finland \\ ${ }^{b}$ Nancy University, CRAN-CNRS UMR 7039, France \\ ${ }^{c}$ Neste Jacobs Oy, P.O. Box 310. FIN-06101, Porvoo, Finland
}

\begin{abstract}
In this paper, a fault tolerant control (FTC) for a dearomatisation process in the presence of faults in online product quality analysers is presented. The FTC consists of a fault detection system (FDI) and a logic for triggering predefined FTC actions. FDI is achieved by combining several process data driven approaches for detecting faults in online quality analysers. The FTC exploits the diagnostic information in adapting a quality controller (MPC) to the faulty situation by manipulating tuning parameters of the MPC to produce both proactive and reactive strategies. The proposed FTC was implemented, tested offline and validated onsite at the Naantali oil refinery. The successful testing and plant validation results are presented and discussed.

(C) 2009 Published by Elsevier Ltd.

Keywords

Fault tolerant control, Fault detection, Data-based modelling, Online analysers, Dearomatisation process, Model predictive control
\end{abstract}

\section{Introduction}

In the highly competitive oil refining industry continuous optimization of the production processes is a crucial factor in keeping the operation economically viable. Enhancing process equipment fault detection and handling the related process disturbances with fault tolerant control are essential factors in maintaining production at the optimised level. Traditionally, industrial fault detection systems have been designed using model-based approaches but, currently, several data-based methods have become real alternatives to first principles modelling as a result of recent development of the methods and improved model identification tools. Commonly used data-based regression models include least squares (LS), partial least squares (PLS), multilayer perceptron networks (MLP) and subspace identified state-space (SS) models.

The PLS was introduced by Wold in the late 1970's and its suitability for chemometry was demonstrated by Wold et al. [38]. Ever since its applicability for different types of processes has been improved with a range of extensions and modifications. A recursive algorithm for partial least squares (RPLS) for time varying processes was first introduced in Helland et al. [6], and it has since been enhanced by Dayal and MacGregor [4], Qin [26,27] and Wang et al. [37]. For very large data sets, Lindgren et al. [16] proposed transforming the large data matrix into a smaller kernel matrix, which could then be analysed with PLS (KPLS). Nomikos and MacGregor [20] presented the idea of multi-way PLS (MPLS) to apply PLS to batch processes. Kourti et al. [14] extended MPLS so that information about the initial conditions of the batch can also be used in the model. The MPLS method unfolds the three-dimensional batch process data into two-dimensions and then performs normal PLS. A true n-dimensional PLS algorithm (N-PLS) for 
analyzing multidimensional data was introduced by Bro [3]. Over the years, numerous extensions have been proposed to make PLS applicable for nonlinear processes. Wold et al. [39] introduced quadratic PLS (QPLS), in which nonlinear (quadratic) functions are used to describe the inner relationships between the input score vectors and the latent variables. Other nonlinear versions of PLS (NLPLS) based on artificial neural networks (ANNs) have been proposed by Qin and McAvoy [28] and Malthouse et al. [18]. Wold [40] proposed using splines to achieve NLPLS. Walczak and Massart [35] proposed a modification to the kernel PLS by using nonlinear radial basis functions (RBF) for creating a nonlinear kernel matrix. Bang et al. [2] have taken yet another approach and integrated fuzzy reasoning to PLS (FPLS) to handle nonlinear cases. By exploiting the Takagi-Sugeno-Kang (TSK) fuzzy model, human expertise can also be integrated into the FPLS model.

Data-based modelling methods include also state-space models, which capture the dynamic properties of processes. The system matrices of the models can be identified using process history data with recently developed subspace model identification (SMI) methods. During the last decade a number of different algorithms for SMI have been proposed; canonical variate analysis (Larimore [15]), N4SID (van Overschee and de Moor [33]), MOESP (Verhaegen [34]) and, more recently, a principal component analysis (PCA) based method by Wang and Qin [36]. The similarities between the different algorithms are studied in van Overschee and de Moor [32], in which a unifying theorem for combining the three algorithms is also proposed. For detailed descriptions of the SMI methods, see e.g. van Overschee and de Moor [33].

Previously described methods have linear foundations and nonlinear processes need different kinds of approach. Commonly, nonlinear processes are modelled with artificial neural networks (ANNs). The most well known type of ANN is the multilayer perceptron network (MLP), that is a multilayer feedforward network typically trained with the supervised back-propagation learning algorithm. According to a study carried out in 1995, 81.2\% of all ANN-based applications utilised the MLP structure (Haykin [5]). A large number of successful industrial applications of these data-based monitoring methods and ANNs have been reported (e.g. Komulainen et al. [13] and Jamsa-Jounela et al. [10] and Kampjarvi et al. [12]) and reviewed, e.g. by Isermann and Ball [8] and Meireles et al. [19].

In this paper, a method for utilising FDI information in fault tolerant process control is introduced in the form of a combined FDI-FTC system for a dearomatisation process. An FTC logic for adapting the tuning parameters of an MPC quality controller in response to a detected analyser fault is also presented. The developed system was tested offline with a simulated process and the real process, and validated online in the real process environment at the Neste Oil Oyj Naantali refinery. The results are presented and discussed. The paper is organised as follows: Section 2 is dedicated to introducing the dearomatisation process. In Section 3, the structure of the implemented FTC is presented. The section also includes descriptions of the proposed FDI and FTC algorithms. Creating the process data driven FDI models is described in Sections 4 and 5 is dedicated to present and analyse the onsite validation results of the FTC system. The paper ends with the conclusions in Section 6.

\section{Dearomatisation process and problem assessment}

Dearomatisation processes are widely used in the oil refining industry. These processes are used to remove aromatic compounds in the feedstock by hydrogenation. The Naantali process is mainly used to remove aromatics from solvents. The dearomatised solvent products have a wide range of quality requirements similar to those of other petroleum oil products. Vaporisation properties are important technical characteristics of the solvent end products and, for this reason, the quality specification of solvents includes minimum requirements for the initial boiling point (IBP) and the flashpoint (FP). The blending characteristics of the initial boiling point and flashpoint are highly nonlinear, making off-spec correction very difficult or even impossible. This makes product quality control particularly important, and this is further escalated by the large volumes of the product tanks. Contaminated products need to be either 
reprocessed or downgraded to less valuable diesel oil. The Naantali process consists of two trickle-bed reactors with packed catalyst beds, a distillation column, a filling plate stripper, several heat exchangers and separation drums, and other unit operations. A simple process diagram of the dearomatisation process at the Naantali refinery is presented in Fig. 1.

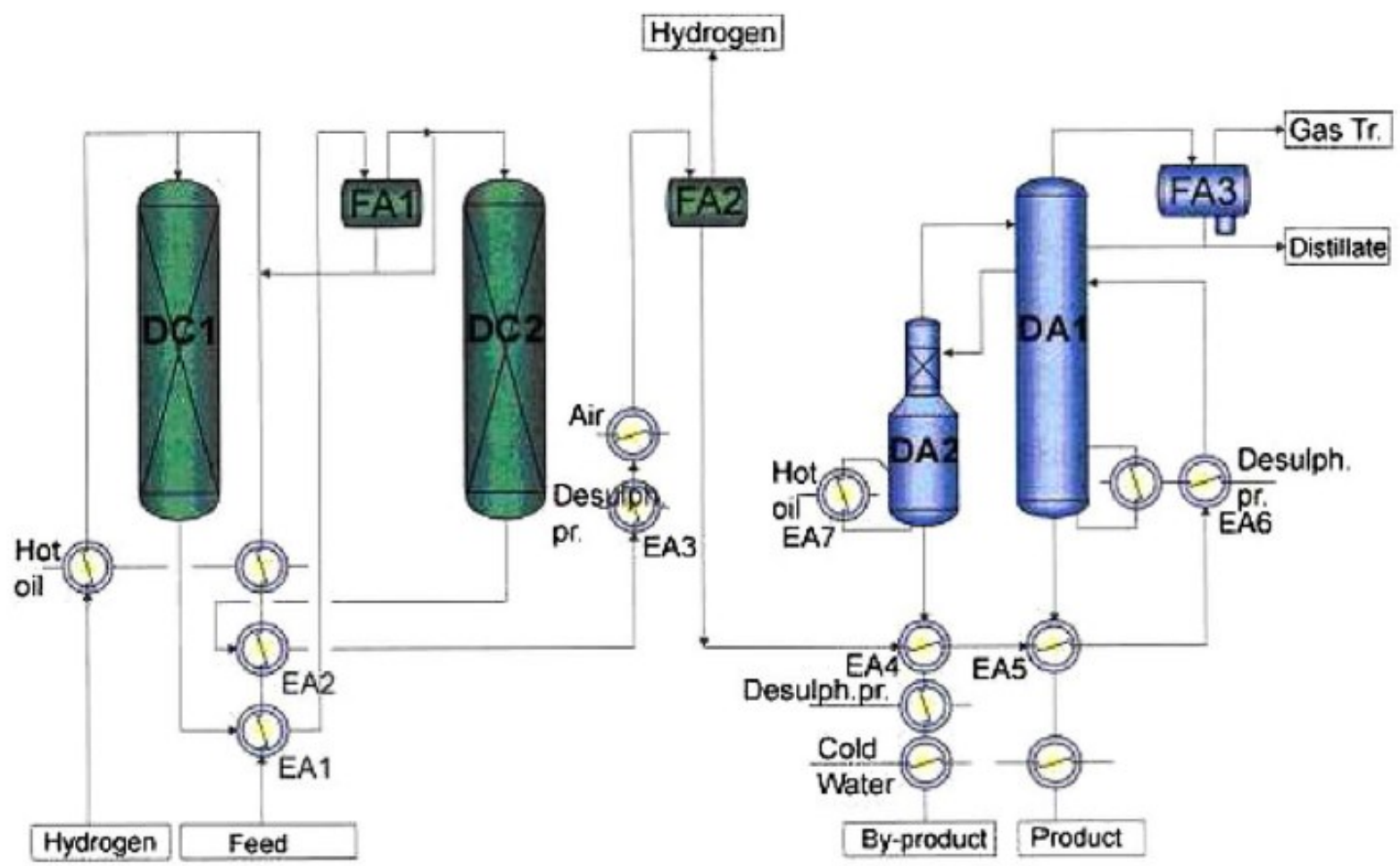

Fig. 1. Dearomatisation process at the Naantali refinery

The quality control of the process is achieved with a model predictive control (MPC) application. In the distillation part of the process, the temperature of the feed is controlled by means of the flow from the desulphuration process to heat exchanger EA6. The reflux ratio of the distillation column is controlled by adjusting the reflux flow rate. The pressure in the column is controlled by an overhead vapour control valve. The operating conditions of the distillation column are controlled by adjusting the flow rate to the reboiler. The highest quality control priority of the MPC in this application is to meet the IBP and FP specifications. Both IBP and FP are measured by online process analysers. The distillation analyser operates on the principle that a product sample is heated up at constant rate while, at the same time, the volume of the evaporated fraction is monitored. The temperatures at which the volume starts to increase, and the temperatures when the volume has reached $5 \%, 10 \%, 50 \%, 90 \%, 95 \%$ of the volume of the sample and the final boiling point of sample, are recorded and given as the outputs of the analyser. The values become available one after another, with several minutes intervals, as the evaporation process progresses. The most significant characteristic of the distillation analyser is the long and variable cycle time of about $40 \mathrm{~min}$ between the subsequent outputs. The flashpoint analyser heats a liquid sample in an open-air container. An electric spark is generated periodically above the container, and the temperature at which the mixture of air and evaporated sample ignites is the flashpoint temperature. In contrast to the distillation analyser, the analysis cycle is relatively short, only about $2 \mathrm{~min}$. Because the analysis results are used by operators and as feedback by the MPC controller, it is important that the analysis results are reliable. Even slowly developing faults in the analysers should be quickly detected and isolated in order to minimise the damage 
risk. Another characteristic of the process is the variability of the feed rate and composition because several feedstock types with clearly distinctive properties are used to produce different grades of solvent.

The dearomatisation process uses an adaptive multivariable MPC for stabilising and optimising control of the large number of MPC variables. Two controlled variables (CV) are of interest in this case: viz. the IBP and FP. The most important manipulated variable (MV) affecting these CVs is the temperature of the distillation column. Other MVs of interest include the column pressure, total process feed flow rate, reflux rate, distillate flow rate, sidedraw rate and column feed preheat temperature. Model predictions are used as feedback in the MPC when no analyser outputs are available during the analyser cycle. Parameters of the distillation prediction models are automatically adjusted online by the MPC application to match the outputs of the online analysers.

The two tuning parameters that are utilised in the FTC are the filtering factor, used with IBP, and the deadband, used with FP. The filtering factor is a parameter for adjusting the importance of the analyser output feedback when updating the MPC predictions. For the almost continuous FP signal, MPC sensitivity to new feedback values is adjusted by manipulating the deadband parameter defining the limit for the smallest deviations of FP from the setpoint considered in control.

The Naantali dearomatisation process MPC uses the online analyser results as feedback from the process to control the IBP and FP. In case the analyser results deteriorate slowly from the true value due to a developing fault, the MPC continues to control the process, but now into new, non-optimal, operating conditions as a result of the faulty feedback. The consequences for too high analyser faulty readings can be very severe because the feedback controls may drive the process to make off-spec product. Challenges in fault detection are the infrequent outputs of the analysers, the clearly separate operating points related to different product grades, and the slow dynamics and long delays of the nonlinear process. The long delays between the distillation analysis results also provide challenges for the FTC, since the corrective actions often have to be made before the faults are detected with full confidence. For this reason, the FTC actions must be such that their effects on the process are reversible in case a fault indication later turns out to be false.

\section{Structure of the FTC and the testing environment}

\subsection{Structure of the FTC}

Once a process fault has been detected and isolated, one way to prevent system deterioration is to develop a controller that has the capabilities needed to compensate for faults. Fault tolerant control systems are characterised by their capabilities to retain performance close to the nominal desired performance after the occurrence of a fault. The accommodation capability of a control system depends upon many factors, such as the severity of the failure, the robustness of the nominal system, the actuator redundancy etc. One classical way of achieving fault tolerant control relies on supervised control where an FDI unit provides the information about the location and time of the occurrence of any fault. Faults are compensated via an appropriate control law triggered according to the diagnosis made by the system. Although FTC is a recent research topic in control theory, the idea of controlling a system that deviates from its nominal operating conditions has been investigated by many researchers. Various approaches for fault tolerant control have been suggested in the literature, e.g. Polycarpou and Helmicki [23], Rausch [29], Patton [22], Ball et al. [1] and Noura et al. [21]. The passive approach makes use of robust control techniques for ensuring that a closed loop system remains insensitive to certain faults. In the active approach, a new set of control parameters is determined in order for the faulty system to reach the nominal system performance while, at the same time, taking into account other criteria such as safety with respect to both failure of the control system and faults in the components. Active FTC strategies utilising model predictive control (MPC) have been studied by e.g. Maciejowski [17], Pranatyasto and Qin [25] and Prakash et al. [24]. More recently 
Jarvinen et al. [11] showed that the inherent accommodation properties of model predictive control (MPC) can readily be exploited to implement different types of FTC strategies, providing the necessary FDI information is available. The concept has been elaborated further in Sourander et al. [30].

The FTC system introduced in this paper consists of two main parts: fault detection and fault tolerant control, acting on the MPC. The FDI system is responsible for detecting faults in the online quality analysers of the dearomatisation process. Information provided by the FDI is given to the FTC, which executes fault tolerant actions according to a predefined triggering logic. The FTC actions affect the tuning parameters of the MPC and are thus realisedwith the MPC and lower level controllers (see Fig. 2).

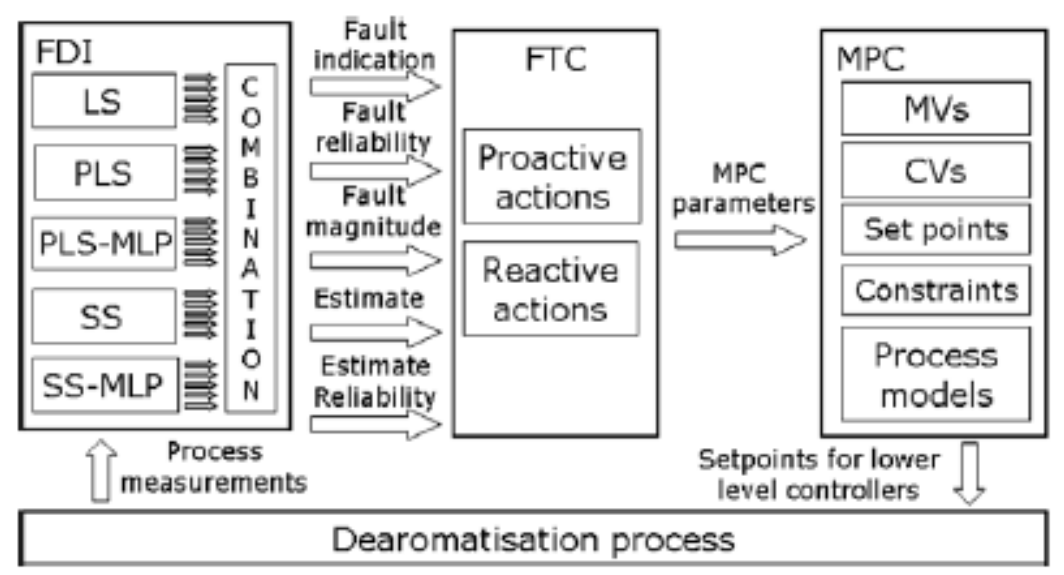

Fig. 2. Structure of the FDI/FTC system

The FDI system is based on four different data-driven FDI techniques: least squares (LS), partial least squares (PLS), multilayer perceptron (MLP), and subspace model identification (SMI), and their combinations. In addition to providing estimates of analyser results and giving fault indications, the FDI provides FTC with information about the reliability of fault indications, the magnitude of detected faults and the reliability of the estimates. The values are derived separately with the different methods and are combined to give the final FDI results.

Fault detection of all the models is based on comparison of the estimated and real analyser outputs. The residual signal is analysed with a modified version of the Page-Hinkley algorithm (original algorithm, Hinkley [7]). The equations for detecting a positive deviation are given in (1)-(3), and similar equations are used to detect negative deviations.

$U_{0}=0$

$U_{k}=U_{k-1}+y_{k}-\mu_{0}-\frac{v}{2}$

$U_{k}=0$ if $U_{k}<0$

where $U$ is the cumulative error sum, $y$ the analysed signal, $\mu_{0}$ the mean of $y \frac{v}{2}$ the minimum size of a fault that is possible to detect with this algorithm, and $k$ a time index. It is noted that the Page-Hinkley algorithm applies to signals with a zero mean, which is not the case here due to model uncertainties. In order to be robust against model uncertainties leading to a non zero mean residual, the value of $\mu_{0}$ is determined online 
for different operation points when the system is running under steady state operating conditions after changes in the feed product. A fault in the analysed signal is detected when

$U_{k} \geq \lambda$

where $\mathrm{k}$ is a threshold for fault detection. The first modification to the algorithm is setting the cumulative sum value at zero when there is no fault ((3)) in order to prevent it from having very large negative values which could cause problems with the computers. The second modification is to clear the cumulative sums after three consecutive good estimations with high estimate reliabilities in order to make the fault status return to the normal state faster after the fault no longer exists.

The developed fault detection method based on cumulative sums is suitable for issuing alarms under normal operation conditions. However, when the feed type is changed, the resulting rapid changes of significant magnitude in the operating conditions may cause the algorithm to trigger false alarms. This is mainly due to two facts: the variable process delays are not exactly known, and the delay compensation is based on approximations of the delays and the time taken by the analysis is not constant. As the estimations for the current analysis results change rapidly, the instant at which the real analyser result becomes available plays a critical role in fault detection. The estimation error caused by comparing the analyser result a few minutes too early or too late with the current estimated values may trigger false alarms. It should be noted that, under normal operating conditions, the product quality does not markedly change in few minutes but, during a feed type change, the distillation and flash point temperatures may increase or decrease significantly. As a consequence, the errors in delay compensation and the varying delays in analysis become problematic. To avoid this problem, all detected faults are suppressed for an adjustable period of time after a feed type change is detected.

The monitoring system indicates a detected fault only when the fault has been detected by all the models.

In the dearomatisation process case where the analyser results are infrequently available, the FTC strategies need to react to the earliest indications of suspected faults in order to maintain the process at the desired state. To support the early FTC actions, the FDI must be tuned to indicate even minor faults that cannot be detected with any certainty. Consequently, the FDI system must provide the FTC with information about the reliability of the fault indications. The reliability of the fault indications by the LS and the rest of the models are determined using two different approaches. The fault reliability based on the PLS, PLS-MLP and SS models is determined using the ratio of cumulative sums and the threshold limits according to (5).

$R_{F}=\frac{\frac{U_{k}}{\lambda}-1}{\sigma}$

where $r$ is a design parameter. If the reliability of the fault is greater than 1 , it is set to 1 . The reliability of the LS-based model is determined by using a sigmoid function

$R_{F_{l s}}=1-e^{-\left(U_{k}-\lambda\right) / \tau}$

where $\mathrm{s}$ is a design parameter. In order to introduce some memory effect, the reliabilities are filtered using a 1st order low pass filter.

The reliability of a detected fault is the minimum of separate reliabilities, based on estimates of the individual models.

The magnitude and direction of detected faults are determined by comparing the estimated analyser outputs with the real ones. The residual is the estimated magnitude of the fault. 
$F_{M}=\hat{y}_{a n}-y_{a n}$

where FM is the estimated magnitude of the fault, $\hat{y}_{a n}$ the estimated value for an analyser, and $y_{a n}$ is the corresponding analyser reading. Estimated magnitudes of faults are determined only when new analyser readings become available.

The fault magnitude is the residual between the average estimate of the analyser outputs and the real ones.

The reliabilities of the estimated distillation and flashpoint temperatures are given in order to indicate the functionality of the FDI system. The reliabilities of the estimates of the PLS models are based on the Hotelling $\mathrm{T}^{2}$ and squared prediction error (SPE) indices [9]. The index values are modified with a sigmoid function:

$y=\frac{1}{1+e^{-a(x-b)}}$

where $y$ is the scaled value, $x$ the original value, and $a$ and $b$ tuning parameters. The parameters have been tuned using the fault free validation data set so that the final reliability in (9) is consistent with the observed estimation error. The final value for the reliability is determined by (2):

$R_{E}=\frac{y_{H T^{2}}+y_{S P E}}{2}$

where $R_{E}$ is the final estimate reliability value and $y_{H T^{2}}$ and $y_{S P E}$ the scaled Hotelling $\mathrm{T}^{2}$ and SPE index values. For combination models consisting of PLS and MLP parts, $R_{E}$ is determined using only the PLS part. The reliability for the estimation is not defined for the LS and SS models.

The combined estimation of the FDI is the average of estimations given by different models. The reliability of the estimated outputs was not directly used in the FTC logic and therefore no combination was needed.

\subsection{FTC}

The implemented FTC scheme (Järvinen et al. [11]) consists of two types of strategy: proactive and reactive. Proactive FTC strategies are associated with low fault detection reliabilities. They aim to minimise the effects of potential faults while causing minimal deterioration of control performance in case the fault detection turns out to be false. MPC retuning is employed to when the reliability of fault detection is moderate. The appropriate MPC parameters are automatically, i.e. without human interaction, retuned to rely less on the analyser measurement as the reliability index of the fault increases. For the distillation analyser the tuning parameter is a simple feedback filter factor, and for the flashpoint it is the deadband. The lower the filter factor, the lower is the significance of the new measurement in the control action. For the deadband it is, in principle, vice versa: the higher a deadband, the lower is the significance of the new measurement. After the MPC is retuned, the future control actions are based on the internal process models of the MPC and the analyzer feedback is used to update the predictions only to a smaller degree. The degree in which the feedback is used is determined on the reliability of the fault indication. Retuning is automatically cancelled if the fault indication turns out to be false.

The reactive FTC strategies are powerful in cancelling the deteriorating effects that the faults have on control before the fault is detected. Temporary feedback deactivation is performed in order to prevent the faulty measurement from affecting control. Once the MPC feedback is deactivated, the controller relies on its internal models while disregarding the faulty feedback in making the predictions for the controlled variables $(\mathrm{CV})$. The second reactive strategy involves shifting the $\mathrm{CV}$ target by the estimated size of a 
detected fault in order to cancel the effects of the faulty feedback on control. This strategy is performed only if the fault may lead to off-spec production, and only after the MPC feedback deactivation has been performed.

FTC strategies are triggered when a small- or medium-sized fault is declared with high enough reliability. Large faults are outside the scope of the FTC as they are detected and handled by the MPC. Proactive retuning is performed when a fault has been detected with moderate reliability. Although the retuning can be a continuous function of the reliability index, in this application three threshold levels (I, II and III, with III being the most severe) were used for easier interpretability of the alarms. Reactive actions are taken when the reliability of a fault is high. In case of abrupt faults, the fault reliability crosses the threshold value as soon as the fault is detected and reactive strategies are immediately executed. With incipient faults, the reliability of fault indication increases gradually due to the progressing fault, and first triggers the proactive strategies and then the reactive strategies. MPC feedback deactivation is always triggered when the reliability index of fault detection reaches a predefined limit value. Target manipulation is performed only if the fault magnitude is negative, leading to a drop in the distillation column temperature that causes a risk of off-spec production. The status of the FTC actions are updated once a minute and, depending on the fault reliability, retuning actions are automatically activated or deactivated, unless reactive FTC strategies have been triggered. When a reactive action is activated, the retunings are deactivated and cannot be activated before the fault has been acknowledged by the operator and removed.

\subsection{Testing environment}

The FTC system has been developed and implemented within the project "Networked Control Systems Tolerant to Faults" (NeCST, EU-IST-004303). The software comprises NeCST global application that carries out the FDI calculations and triggers the FTC actions, and NeCST local application that is integrated within the refinery process control infrastructure and executes the necessary process database access functions. The global and local applications communicate via the refinery network using FTP and XML files to transfer data. The local application provides process data for the global application. It also executes the FTC actions by accessing and modifying the database variables of the MPC according to the instructions it receives form global application. The implemented system was first tested offline with a simulated process and a process control computer, and with real process data and a remote duplicate of the Naantali refinery VMS process computer. The validation of the system was then carried out in an industrial environment at the Neste Oil Oyj Naantali refinery in Finland. The software setups and environments corresponding to testing and validation are illustrated in Fig. 3.

\section{Process data driven FDI and offline tests}

The data used for creating the FDI models were acquired during the winter 2006-2007 from the Naantali refinery, and it covered the operation of the dearomatisation process for a period of 4 months and 1 week. All the main product grades were in production during that period. Values of the variables were 1-min samples, except for the analyser measurements which had sampling frequencies of about $2 \mathrm{~min}$ in the case of FP and about $40 \mathrm{~min}$ in the case of IBP.

The dearomatisation process exhibits long transport delays and slow dynamics and, as a consequence, the values of the recorded variables were time shifted to improve the accuracy of the FDI. The delays were estimated with cross correlation analysis and compensated for by shifting the values of the variables forward in time to match the corresponding analyser result times. After the delay compensation, sections of data representing abnormal operation of the process and/or the analysers were removed. 


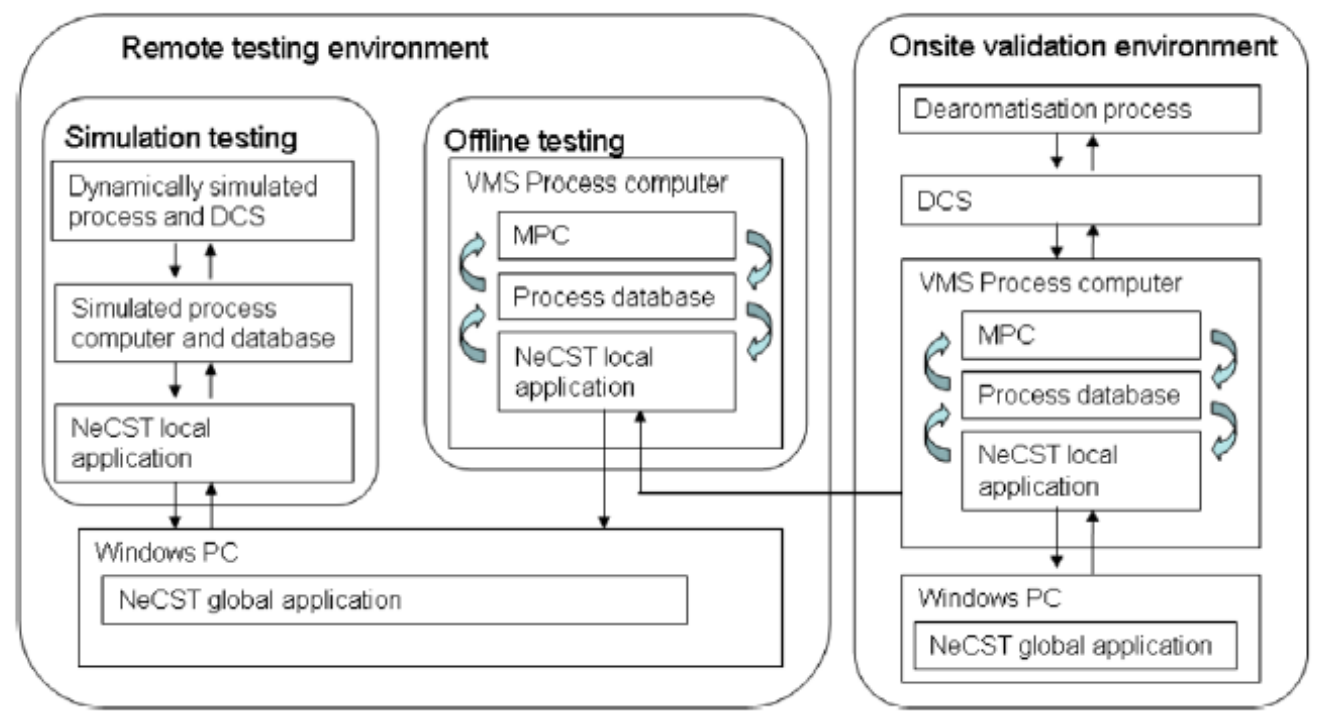

Fig. 3. Offline testing and onsite validation environments

Next, process knowledge and measured variables were used to generate calculated variables describing process specific phenomena. The operational status of the whole process is closely reflected by the state of the distillation column. In turn, the temperature profile and the pressure of the column are strongly influenced by the characteristics of the feedstock. This dependence on the feedstock type makes it challenging to use the temperature measurements as input variables for fault detection. The temperature differences between different trays are, however, less affected by the operating point, and thus offer useful information about the state of the process in all operation conditions and for all product grades. Also, the pressure-compensated temperature is a useful input variable in modelling the different pressure ranges used for different grades. The temperature of the bottom product is useful as such because it is the variable most strongly correlating with the analyser outputs. The temperatures of other parts of the column are better presented as differences over a certain part of the column.

The input variable sets for the FDI methods were determined using three selection methods in succession. First, process knowledge and engineering judgement were used to discard variables that did not describe the behaviour of the analyser outputs or were not consistent for all products. Second, during the correlation analysis of the delay compensation phase, variables with weak correlation with the outputs were also discarded. Third, the final selection was performed with mathematical wrapper type of input variable selection methods; a modified forward selection method and genetic algorithms. The optimal number of the input variables and the input variable set depends on the model type. The number of input variables ranged from three to six.

Finally, to reduce the quantity of the data, only those samples corresponding to the instants when new analyser readings had become available were used. For the distillation analyser models the training set consisted of about 3750 samples, and for the FP models about 75000 samples. Of the training data sets $75 \%$ of the data was used for actual training and $25 \%$ for model evaluation. The data sets were divided so that both the training and the evaluation set consisted data representing all the different operating regions.

\subsection{FDI models}

In order to evaluate the suitability of different process history data-based modelling methods, least squares (LS), partial least squares (PLS), multilayer perceptron (MLP), and subspace identified state-space models (SS) were created. The eight models for FDI that were implemented and evaluated were: LS, PLS and PLS- 
MLP models for IBP and FP temperatures, an SS model for IBP, and an SS-MLP model for FP. The structure of the LS models is expressed as

$\hat{y}(k)=y_{0}+\Delta \hat{y}(k)$

$\Delta \hat{y}(k)=\phi^{\prime}(k) \Theta$

$\phi^{\prime}(k)=\left[\Delta x_{1}\left(k-N_{1}\right), \ldots, \Delta x_{p}\left(k-N_{p}\right)\right]$

$\Delta x_{1}(k)=x_{1}(k)-x_{10}$

$\Theta=\left[\Theta_{1}, \ldots, \Theta_{p}\right]^{\prime}$

where $\hat{y}(k)$ is the output prediction, $x_{i}(k)$ the measured state variables which represent the input of the model, and where $\left[x_{10}, \ldots, x_{p 0}, y_{0}\right]$ designate the operating conditions, $\Theta$ a vector of the parameters, and $N_{i}$ the delays. It should be noted that the input variables introduced in the models were not control input variables, but represented state variables. From this point of view, the models given by (10)-(14) can be considered to be soft-sensor models. LS models had an input variable set of six variables, listed in Table 1.

Table 1

Input variables of the models with estimated delays [min]

\begin{tabular}{|c|c|c|c|c|c|c|}
\hline Variable & IBP & & & $\mathrm{FP}$ & & \\
\hline & LS & PLS & SS & LS & PLS & SS-MLF \\
\hline Column 1 stripping section pressure compensated temp. ${ }^{\circ} \mathrm{C}$ & 60 & 66 & 66 & 60 & $\mathrm{X}$ & 53 \\
\hline Column 1 stripping section temp. ${ }^{\circ} \mathrm{C}$ & 60 & 71 & $\mathrm{x}$ & 60 & 60 & $\mathrm{x}$ \\
\hline Column 1 reboiling temp. ${ }^{\circ} \mathrm{C}$ & $\mathrm{x}$ & 79 & 79 & $x$ & $\mathrm{x}$ & $\mathrm{x}$ \\
\hline Colunm 1 mid section temp. ${ }^{\circ} \mathrm{C}$ & 60 & 75 & 75 & 60 & $\mathrm{x}$ & $x$ \\
\hline Colunm 1 rectifying section temp. ${ }^{\circ} \mathrm{C}$ & $\mathrm{x}$ & 61 & $\mathrm{x}$ & $x$ & $\mathrm{x}$ & $x$ \\
\hline Colunm 1 bottom tray temp. ${ }^{\circ} \mathrm{C}$ & 60 & $x$ & $\mathrm{x}$ & 60 & $\mathrm{x}$ & $x$ \\
\hline Colunm 1 bottom product temp. ${ }^{\circ} \mathrm{C}$ & 60 & $\mathrm{x}$ & $\mathrm{x}$ & 60 & $\mathrm{x}$ & $\mathrm{x}$ \\
\hline Colunm 1 reboiler return temp. ${ }^{\circ} \mathrm{C}$ & 60 & $x$ & $x$ & 60 & $x$ & $x$ \\
\hline Column 1 top pressure (kPa) & $\mathrm{x}$ & 40 & 40 & $x$ & 39 & 39 \\
\hline Column 2 top pressure ( $\mathrm{kPa})$ & $\mathrm{x}$ & $x$ & 41 & $\mathrm{x}$ & $\mathrm{X}$ & $x$ \\
\hline Side product flow rate $(\mathrm{t} / \mathrm{h})$ & $x$ & $x$ & 41 & $x$ & $\mathrm{x}$ & 53 \\
\hline Column 1 heating medium flow $(\mathrm{t} / \mathrm{h})$ & $\mathrm{x}$ & $\mathrm{X}$ & $\mathrm{x}$ & $\mathrm{x}$ & $\mathrm{x}$ & 44 \\
\hline Feed type switch & $x$ & $x$ & $x$ & $x$ & 206 & $x$ \\
\hline Column 1 stripping section delta temp. ${ }^{\circ} \mathrm{C}$ & $\mathrm{x}$ & $\mathrm{x}$ & $\mathrm{x}$ & $x$ & N/A & N/A \\
\hline Colunm 1 rectifying section delta temp. ${ }^{\circ} \mathrm{C}$ & $\mathrm{x}$ & $\mathrm{x}$ & $\mathrm{x}$ & $x$ & N/A & $\mathrm{x}$ \\
\hline Colunm 1 reboiler section delta temp. ${ }^{\circ} \mathrm{C}$ & $x$ & $\mathrm{X}$ & $\mathrm{X}$ & $X$ & $\mathrm{X}$ & $\mathrm{N} / \mathrm{A}$ \\
\hline
\end{tabular}

$\mathrm{X}$ : denotes that the variable is not used in a model.

$\mathrm{N} / \mathrm{A}$ : denotes that no delay compensation is required for the variable.

Partial least squares (PLS) was chosen as an FDI method due to the excellent results reported by Komulainen [13]). The PLS model for IBP was trained with six variables. Of these six variables, five latent variables were used to construct the model. The input variable set of the FP model consisted of five variables which were approximated with three latent variables. The number of latent variables for both models were determined using separate validation data sets.

To address the nonlinearities of the process, the PLS models for IBP and FP were augmented with an MLP network with a single layer of hidden neurons. The MLPs were trained using the Levenberg-Marquardt training algorithm with 50 epochs and the same input data as the PLS models, while the output was the residual between the analyser outputs and the corresponding values estimated by the PLS models. Before training the MLPs, the residual signals were bounded in order to minimise the effects of isolated instants 
with large absolute values on the training process. The PLS parts of the combination models were the same as those described earlier for the IBP and FP. Using separate validation sets covering plant operation for approximately one month, the optimal number of neurons in the hidden layer was found to be five for the IBP model, and four for the FP model.

Although the PLS-MLP models are able to describe both the linear and nonlinear behaviour of the process, they ignore the part of the dynamics of the process that is not completely accounted for by the delay compensation method described earlier. To model the dynamics, SS models were identified for IBP and FP using a subspace identification method (SMI). The SS model for FP was augmented with an MLP model in a similar fashion to that for the PLS-MLP models. The IBP model was created with five variables and consisted of two states. The reduction in the input variable dimension was carried out with PCA. The FP model had two states and was trained with six variables. The MLP part of the combined model had three neurons in the hidden layer.

The input variables for all the methods are presented in Table 1, which also lists the estimated delays between the input and output variables.

\subsection{Offline testing of the estimation capability of the FDI}

In the next project phase, the estimation accuracy of the FDI methods was tested offline with a dynamic chemical engineering simulator. A good fitting of the training data was obtained with all the tested methods. The actual measurement and LS model prediction for the IBP considering a 4 weeks data set, exhibiting changes in the feed product, is presented as an example in Fig. 4).
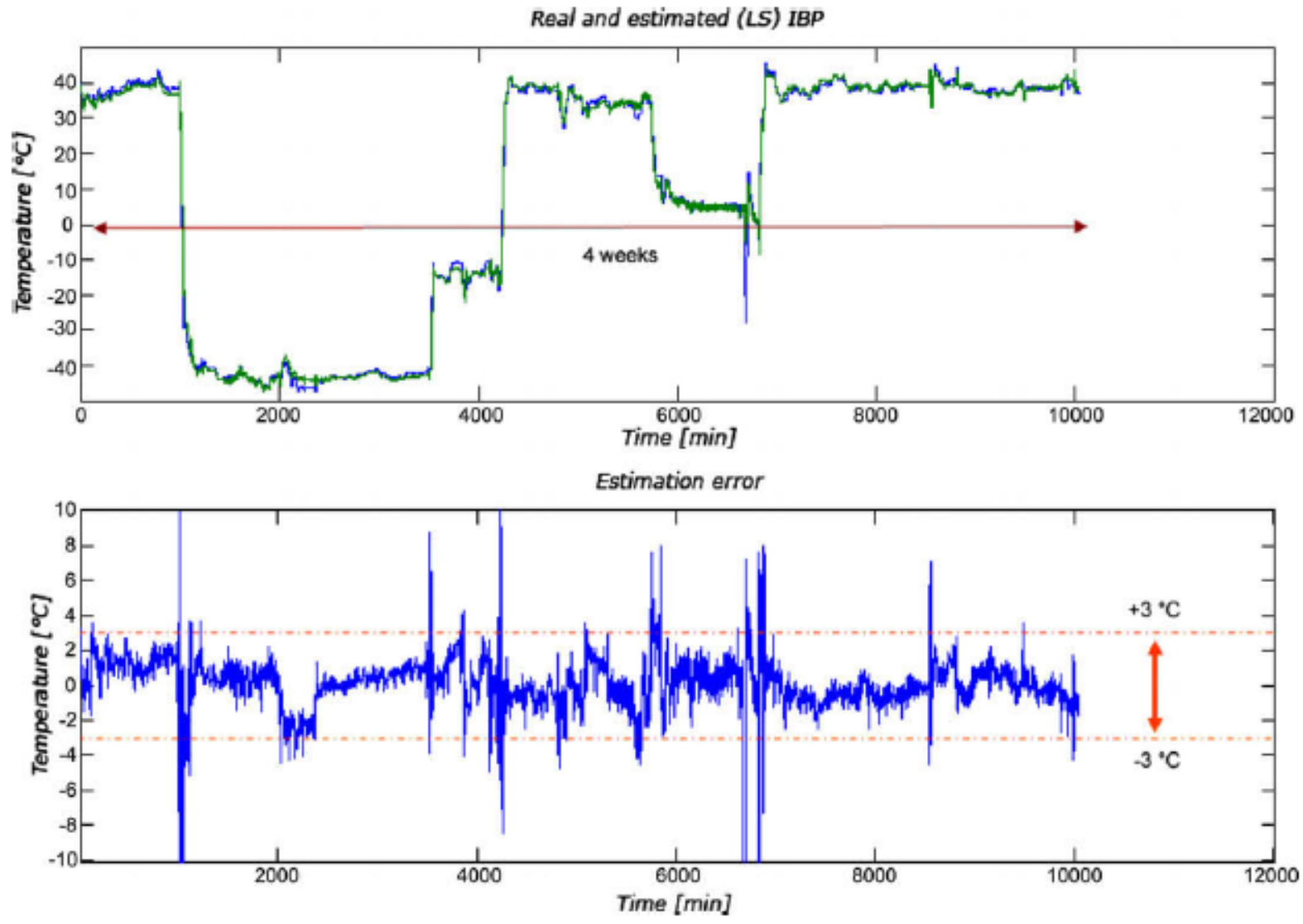

Fig. 4. Identification of the LS model for IBP 
The estimation error illustrated in the figure is zero mean and belongs to the interval $1 / 2 \_3 \_\mathrm{C}$ p $3 \_\mathrm{C}$, except for a few outliers that mainly occurred during the transition periods after feed stock changes. It is noted that a bias has been added to the presented values of IBP as it is a policy of the Naantali refinery that no real data are published. The testing has been described in detail in Sourander et al. [31].

For model testing, a set of one week data that was not used in the model identification was considered. The results in Fig. 5 clearly show that the estimation error is no longer zero mean. Nevertheless, for a given feed product the estimation error apparently has a constant mean. The important conclusion is that this kind of model can be used for fault detection, considering the low prediction errors. However, special attention should be paid to the robustness against uncertainties such as outliers or model uncertainties due to feed product changes.
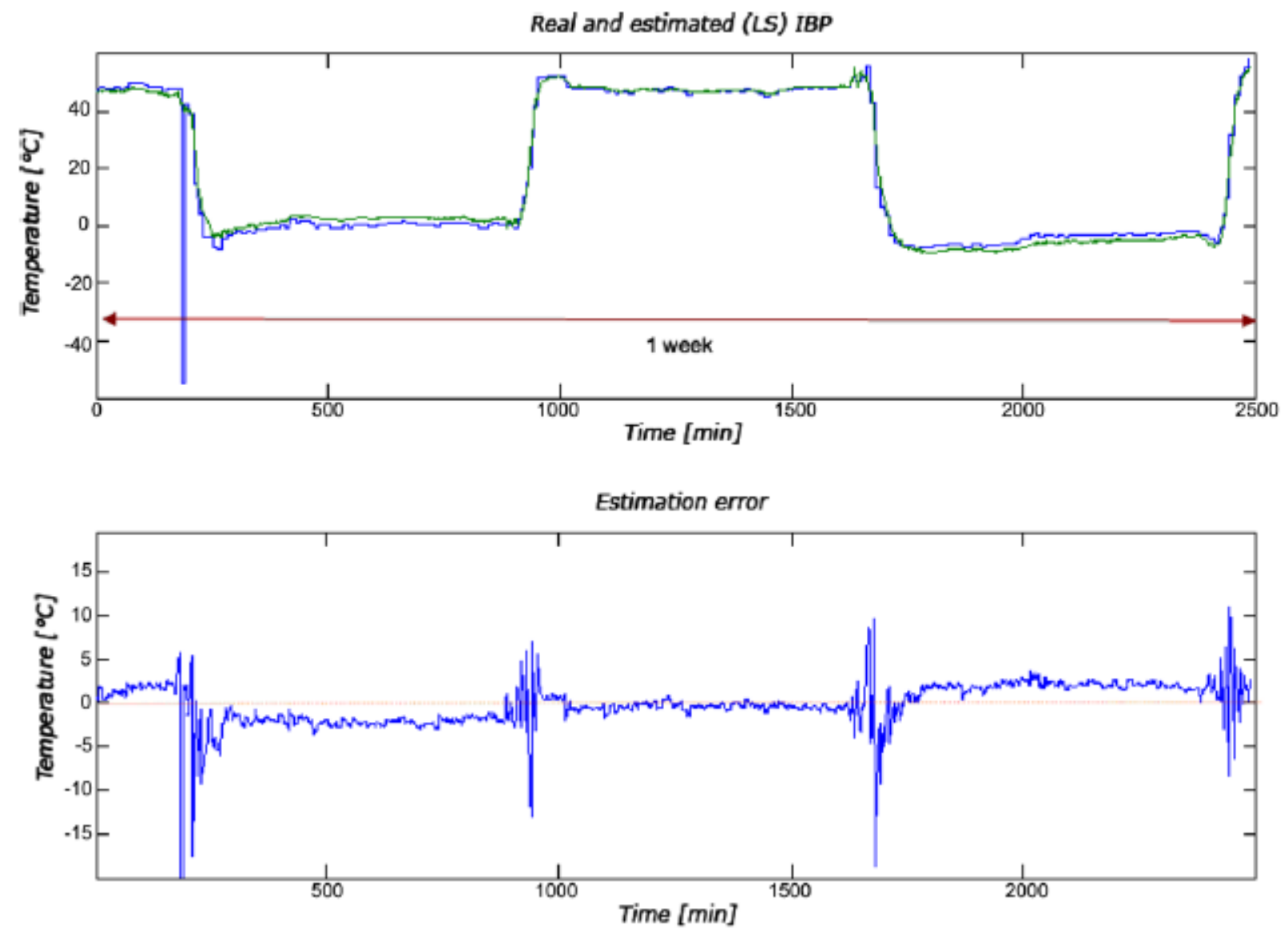

Fig. 5. Offline test of the estimation accuracy of the LS model for IBP

\section{Online validation of the FTC system at the refinery}

The developed fault tolerant control system was validated onsite at the Naantali oil refinery during May 2007. During the validation period, four different feed types were used in seven runs. For the purpose of validating the FDI/FTC performance, the IBP and FP analyser results were manipulated in order to produce artificial analyser faults similar to naturally occurring ones. Abrupt faults were produced by introducing a single bias to the analyser values, whereas simulating drift type faults required the introduction of a ramp function. 


\subsection{Fault detection}

The IBP and FP temperatures were estimated with the different methods. The analyser outputs during the validation period, and the corresponding estimation errors of the different methods, are shown in Figs. 6 and 7. Most of the estimations obtained with all the methods were within an acceptable estimation error limit of $3 \ldots$. Larger deviations from the real analyser values were mainly encountered during the transition periods after the feed type changes. The distributions of estimation errors are shown in Fig. 8, and the root mean squared error of the estimations (RMSE) in Table 2. According to the distribution of the estimation errors, the three methods having the highest percentage of the estimations within 3 C of the real IBP were the combined PLS-LS, PLS and PLS-MLP. The PLS-LS values are averages of the separate PLS and LS models. In contrast, the RMSE of the combined PLS-LS was notably higher than that of PLS-MLP, PLS and SS. In the FP case, the most accurate method was PLS-MLP with more than $97.2 \%$ of the estimates under the target limit of 3 C and an RMSE value of 2.15 C. The next best methods were PLS and combined PLS-LS. Overall, the approach based on PLS and MLP gave the most accurate estimations, followed by PLS and combined PLS-LS. Statespace and LS approaches were notably less accurate.
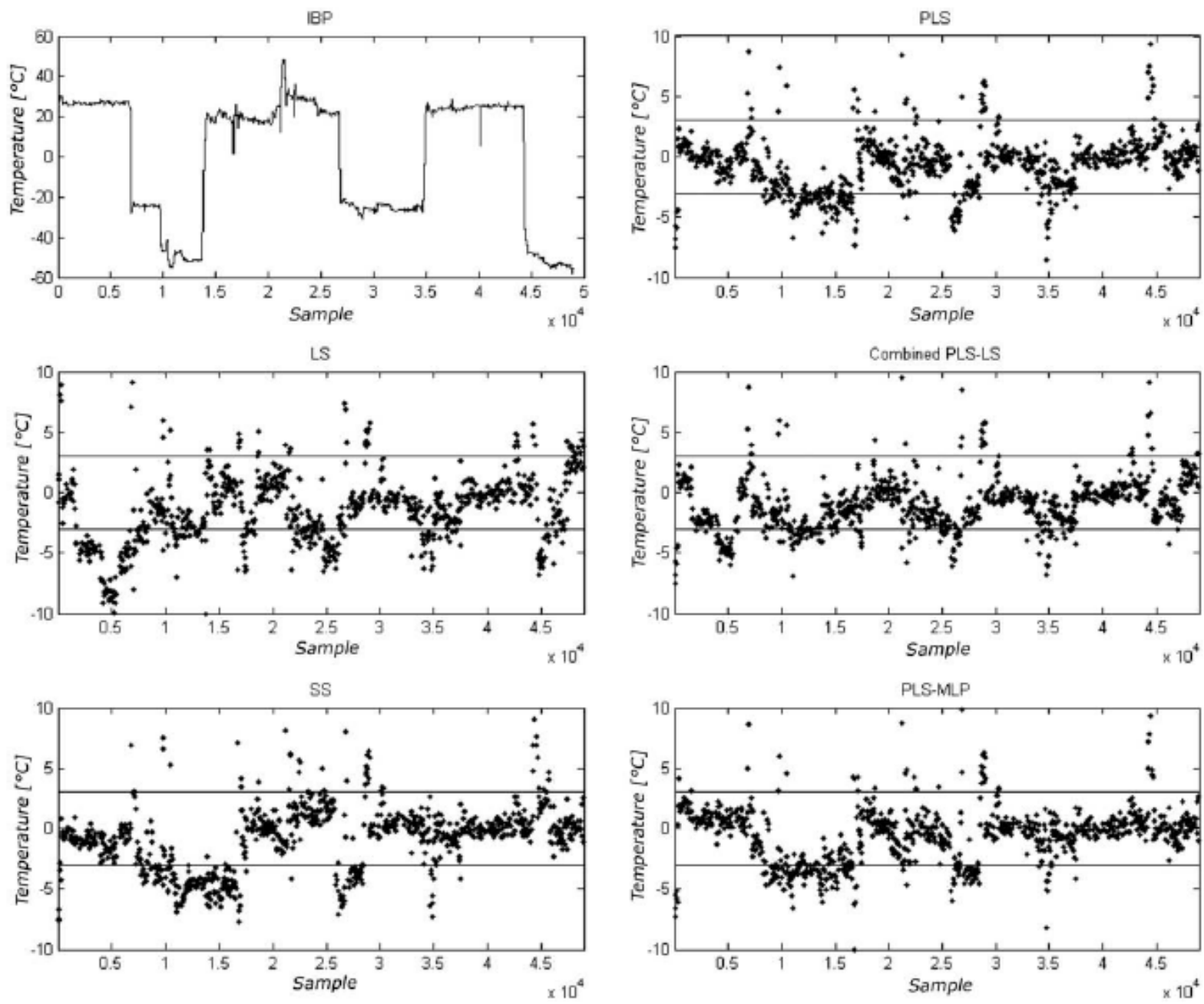

Fig. 6. Real IBP temperatures and estimation errors

During the validation period twelve analyser faults were introduced. The faults included one abrupt and three drifting faults in the distillation analyser, and two abrupt and six drifting faults in the flashpoint 
analyser. The performance evaluating emphasized the detection of drift type faults that have been common in the past and are very difficult for the operators to detect. The fault detection parameters $\mathrm{m}$ and $\mathrm{k}$ of the modified Page-Hinkley algorithm were found useful in tuning the fault detection system onsite. The ultimate goal of the tuning was to find suitable values for $\mathrm{m}$ and $\mathrm{k}$ so that the FDI/FTC system is neither too sensitive nor too indifferent to faults: the system should be able to detect difficult, small-sized incipient faults without triggering false alarms. The tuning was performed by hand by estimating a fault size $ð \mathrm{~m}=2 \mathrm{P}$ that the system should be able to detect, and by choosing a suitable threshold value for fault detection $ð \mathrm{kP}$. It was possible to examine the Page-Hinkley cumulative sum U online. This facilitated the tuning of the fault detection parameters. Examples of fault indication and the reliability information are presented in the next section related to the FTC.
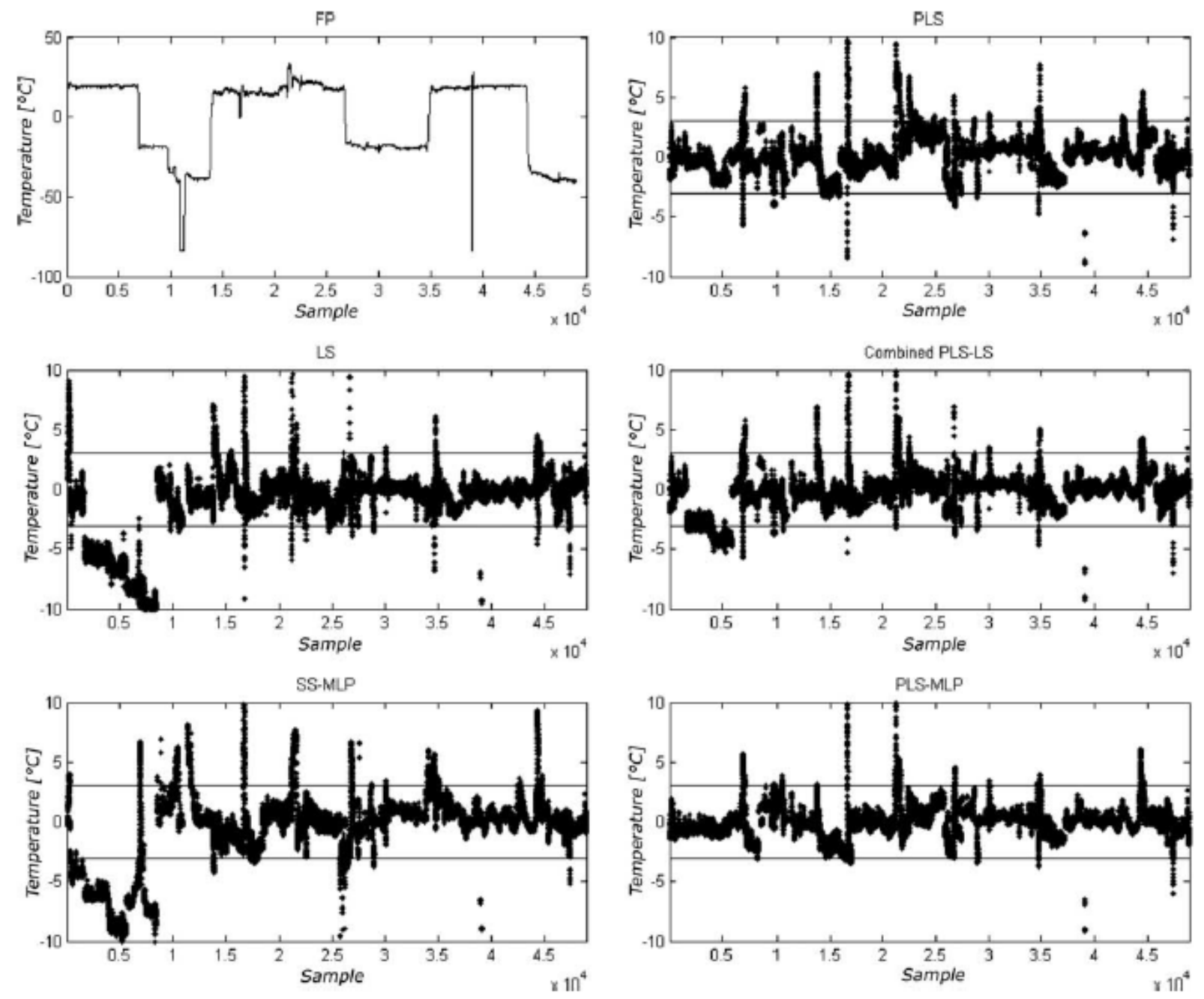

Fig. 7. Real FP temperatures and estimation errors

Table 2

RMSE of IBP and FP estimates

\begin{tabular}{lllllll}
\hline RMSE $\left({ }^{\circ} \mathrm{C}\right)$ & LS & PLS & PLS-MLP & SS & SS-MLP & Combines PLS-LS \\
\hline IBP & 6.99 & 3.22 & 3.18 & 3.67 & - & 4.48 \\
FP & 3.85 & 2.64 & 2.15 & - & 3.56 & 2.54 \\
\hline
\end{tabular}



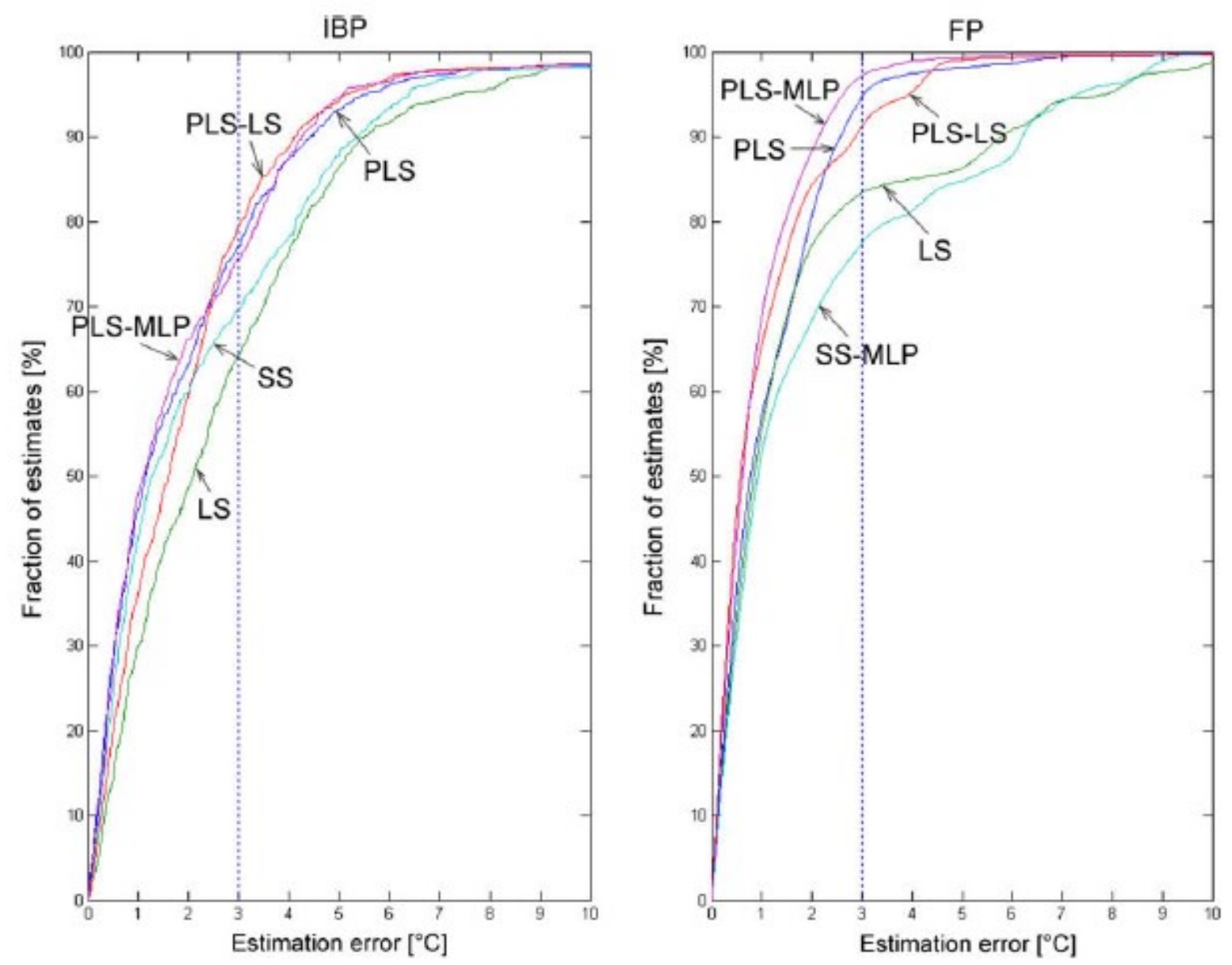

Fig. 8. Distribution of estimation errors, IBP (left) and FP (right)

\subsection{Fault tolerant control}

The FTC strategies tested previously offline (Sourander et al. [30,31]) were taken into use at the refinery. In the twelve introduced fault cases, both the FDI and FTC performed as expected, and all the faults were detected. The performance was very similar in all cases, and in the following the evolution and functioning of two typical fault case examples are discussed in more detail. The first example is an incipient drift type fault upwards in the distillation analyser IBP, and the second is an incipient drift type fault downwards in the FP analyser. These two examples illustrate the versatility and applicability of the FTC strategy in two substantially different cases with differing fault directions, detection delays and analyser cycle times. Note that, in the following, the temperatures are biased by a constant in order to respect the plant policy not to publish actual production data.

In the first example, the analyser result for IBP is artificially increased by 4.5 C in four steps. The resulting elevation in the IBP temperature remained relatively small as the MPC controls the process to keep it close to the desired level. The controller uses the distillation column temperature to control the initial boiling point and, under closed loop operation, a fault in the initial boiling point result is most prominently reflected in the value of this MV. Seventy-two minutes after the fault is introduced, the FDI gives a fault indication with moderate reliability and a proactive FTC strategy of MPC retuning is triggered. The reliability of the fault indication increases when the next distillation analyser result for IBP becomes available, and the reactive FTC strategies of MPC feedback deactivation and CV target manipulation are triggered. As a result of these FTC actions, the drop in the value of the column temperature MV caused by the faulty analyser 
feedback is almost completely cancelled. The progress of the relevant process measurements and the outputs of the FDI system during the upward incipient fault are illustrated in Fig. 9. In the figure, the IBP and column temperature (MV) are shown in addition to the information provided by the FDI, the estimated IBP, the fault indication and the reliability of the fault indication.
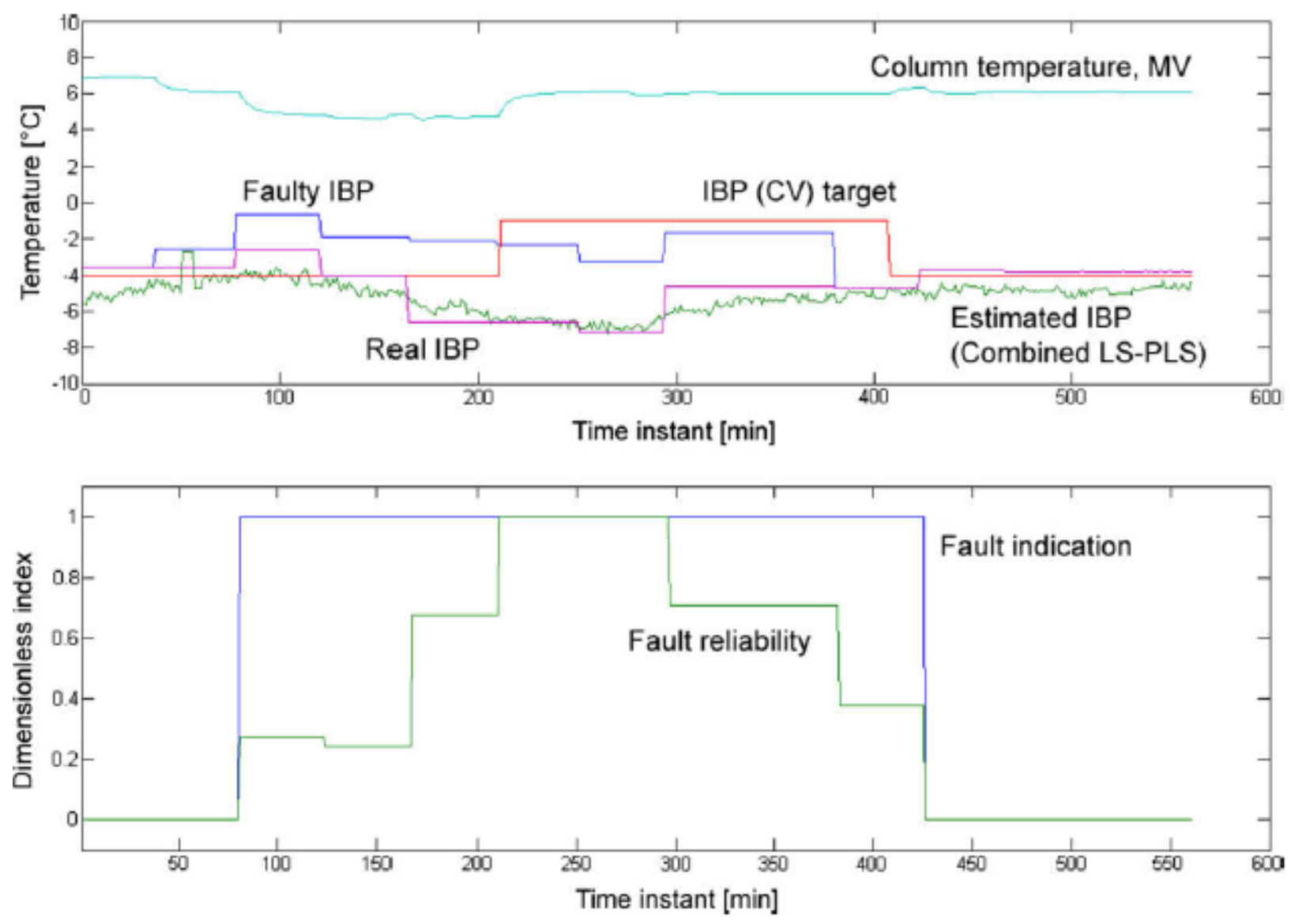

Fig. 9. Distillation analyser upward fault

The second example is a downward incipient fault in the flashpoint analyser result. During the test, the MPC was controlling the flashpoint of the solvent product. This case also illustrates the classical problem of detecting sensor faults in a closed loop, because the controller maintains the value of the faulty sensor at a constant level, thus masking the faults. To introduce a fault, the analyser result was manipulated in 24 steps of_0.1_C in magnitude conducted at five minute intervals. The fault was detected at instant $153 \mathrm{~min}$ when its magnitude was 2.3 C. After the initial fault indication, appropriate FTC strategies were triggered according to the fault indication reliability index. As the reliability of the fault increased gradually, all the retuning actions (I, II and III) were triggered one after another. Finally, the reactive FTC strategy of MPC feedback deactivation was triggered. Since the fault was downward, there was no CV target manipulation. The effects of the FTC are reflected in the values of the distillation column temperature (MV), which is the main lever that the MPC uses to control the solvent flashpoint. Before the fault is detected, the MPC increases the value of MV to achieve the setpoint of 4 _ C. Once the fault is detected, the MPC relies less on the analyser outputs and gradually ceases making changes to the MV, while also stabilising the real FP value to 4 _C. After the fault has been fixed at instant $256 \mathrm{~min}$, the fault indication is removed $12 \mathrm{~min}$ later. The value of the MV returns to its original level $8 \mathrm{~min}$ after the analyser has returned to the normal operation state according to FDI. Fig. 10 shows the relevant process measurements and FDI information 
associated with this case. The upper figure shows the real, faulty and estimated FP temperatures and the values of the MV. The lower part of the figure shows the fault indication and the reliability of the fault.
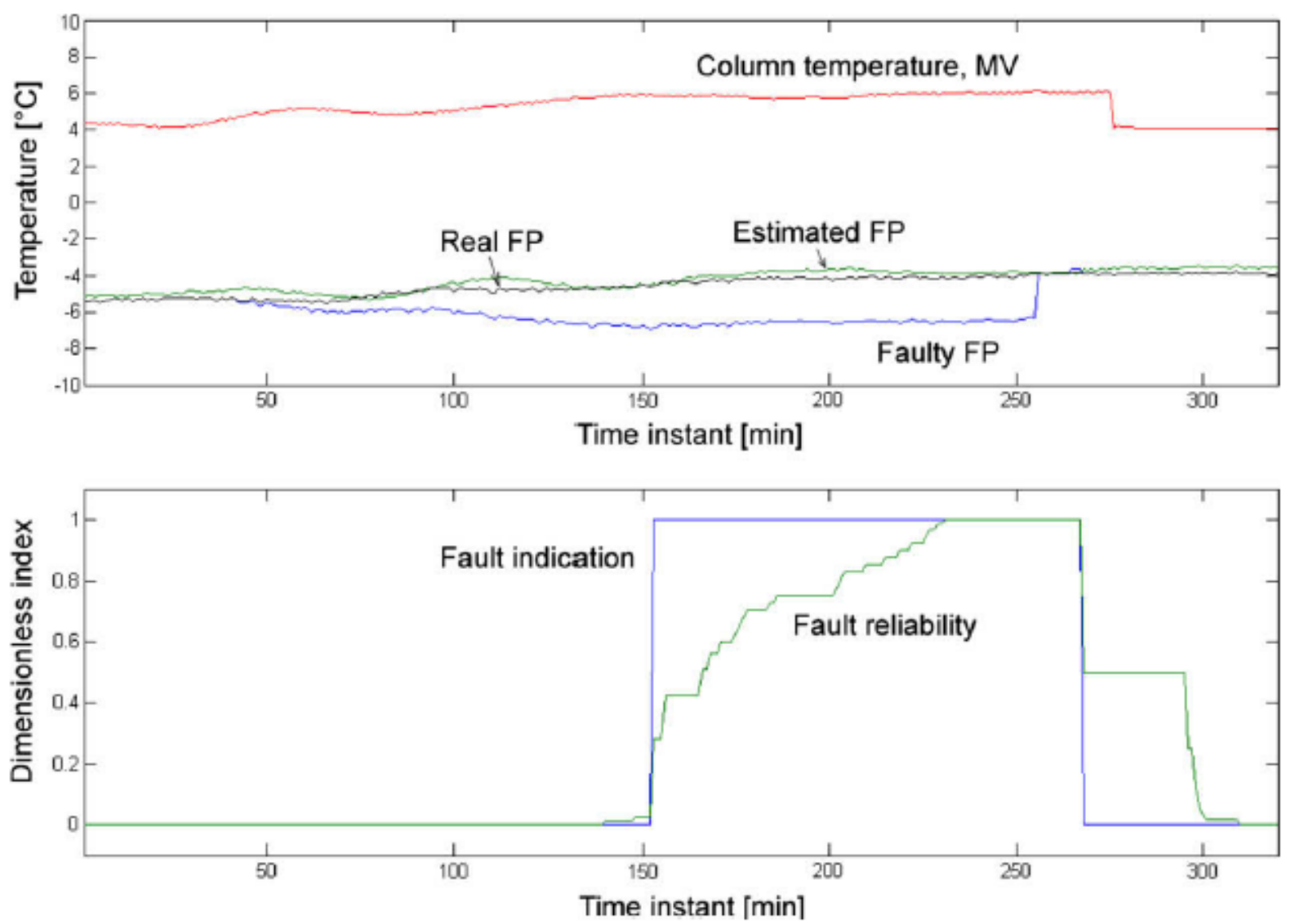

Fig. 10. Flashpoint analyser downward fault

\subsection{Analysis of the results and discussion}

The validation results of the FDI/FTC system presented in the previous section prove that the system worked as planned and as required by the refinery. The performance of the system conformed in all respects to the specification and expected behaviour, and the FDI/FTC interactions with the process were as expected. The impact of proactive and reactive FTC strategies was, for the most part, found to follow the results obtained from dynamic simulations prior to the actual refinery validation.

The acquired validation results also show that incorporating the solvent dearomatisation unit model predictive controller with the tested FDI/FTC system allows the inherent prediction, dead time compensation, and accommodation properties of MPC to be taken into use in order to achieve fault tolerance towards sensor faults. Accurate FDI continues to be the most important module of FTCaugmented MPC. Without correct fault detection information, the FTC capabilities of such a system are limited. The flexibility introduced by using the reliability index as an indicator for triggering different FTC actions on demand is of high value because this allows the use of FTC strategies of different severity levels. Proactive FTC strategies influenced the process only moderately during the tests, but bought more time for an improved FDI analysis and alerted the operators to watch out in case there was a fault in one of the analysers. In cases where the fault detection was false, the proactive FTC strategies were simply cancelled without any serious consequences. On the other hand, in cases where the fault indication was justified, the 
deterioration induced in control by the fault was far less severe than if no proactive measures had been taken. The validation tests also demonstrated the great potential of reactive FTC strategies for maintaining the product qualities at desired levels in the presence of analyser faults. Especially the CV target shift with temporary $\mathrm{CV}$ feedback deactivation is a safe, powerful, and reliable strategy. Off-spec production caused by faulty feedback could be avoided in all the studied cases using these strategies.

\section{Conclusions}

A control strategy tolerant to faults in online quality analysers has been developed for the Naantali refinery dearomatisation process. The developed system consists of FDI and FTC parts. The FDI is based on simultaneously using several process data driven methods. Besides indicating a fault, the FDI system also estimates the reliabilities of the indications, thereby providing means to easily tune the sensitivity of the FTC strategy. The FTC logic exploits two types of action, proactive and reactive. Proactive actions are performed when faults are suspected and their effects on the control are easily reversed in the case of false alarms. Reactive actions manipulate the control with severe consequences and are only performed when faults are detected with high reliability. The developed system has been tested offline with a simulated process and the real process, and validated onsite at the Naantali refinery. The results were presented and discussed. The analysis of the results shows that the FTC strategies enabled by an accurate FDI system are capable of preventing off-spec production and quality giveaway.

\section{Acknowledgements}

The authors gratefully acknowledge the personnel at the Naantali refinery and of Neste Jacobs for their support. This research has been carried out in connection with the EU research project: NeCST - Networked Control Systems Tolerant to faults (IST-004303). The project is partially funded by the European Union. The support is gratefully acknowledged.

\section{References}

[1] B. Ball, M. Fisher, D. Fussel, O. Nelles, R. Isermann, Integrated control diagnosis and reconfiguration of a heat exchanger, IEEE Control Syst. Mag. 18 (3) (1998) 52-64.

[2] Y.H. Bang, C.K. Yoo, I.-B. Lee, Nonlinear PLS modeling with fuzzy inference system, Chemom. Intell. Lab. Syst. 64 (2003) 137-155.

[3] R. Bro, Multiway calibration, multilinear PLS, J. Chemom. 10 (1996) 47-61.

[4] B.S. Dayal, J.F. MacGregor, Recursive exponentially weighted PLS and its applications to adaptive control and prediction, J. Process Control 7 (1997) 169-179.

[5] S. Haykin, Neural Networks: A Comprehensive Foundation, second ed., Prentice Hall, 1995. p. 842.

[6] K.B. Helland, H.E. Berntsen, O.S. Borgen, H. Martens, Recursive algorithm for partial least squares regression, Chemom. Intell. Lab. Syst. 14 (1992) 129-137.

[7] D.V. Hinkley, Inference about the change point from cumulative sum tests, Biometrika 58 (1971) 509523.

[8] R. Isermann, P. Ball, Trends in the application of model-based fault detection and diagnosis of technical processes, Control Eng. Practice 5 (1997) 709-719.

[9] J.E. Jackson, A User's Guide to Principal Components, Wiley \& Son's, New York, 1991. p. 569.

[10] S.-L. Jamsa-Jounela, M. Vermasvuori, P. Endn, S. Haavisto, A process monitoring system based on the Kohonen self-organizing maps, Control Eng. Practice 11 (2003) 83-92.

[11] E. Jarvinen, M. Sourander, T. Liikala, Evaluation of different FTC strategies for a refinery process, in: R. Patton, A. Casavola, E. Rondeau (Eds.), Proceedings of the Second NeCST Workshop, Rende, 2006. 
[12] P. Kampjarvi, M. Sourander, T. Komulainen, M. Nikus, N. Vatanski, S.-L. Jamsa-Jounela, Online analyser validation and process fault diagnosis for ethylene cracking process under MPC feedback, Control Eng. Practice 16 (2007) 1-13.

[13] T. Komulainen, M. Sourander, S.-L. Jamsa-Jounela, An online application of dynamic PLS to a dearomatization process, Comput. Chem. Eng. 28 (2004) 2611-2619.

[14] T. Kourti, P. Nomikos, J.F. MacGregor, Analysis monitoring and fault diagnosis of batch processes using multiblock and multiway PLS, J. Process Control 5 (1995) 277-284.

[15] W. Larimore, Canonical variate analysis in identification, filtering, and adaptive control, in: Proceedings of the 29th IEEE Conference on Decision and Control, Honolulu, 1990, pp. 596-604.

[16] F. Lindgren, P. Geladi, S. Wold, The kernel algorithm for PLS, J. Chemom. 7 (1993) 45-59.

[17] J.M. Maciejowski, Modelling and predictive control: enabling technologies for reconfiguration, Ann. Rev. Control 23 (1999) 13-23.

[18] E.C. Mathouse, A.C. Tamhane, R.S.H. Mah, Nonlinear partial least squares, Comput. Chem. Eng. 21 (1996) 875-890.

[19] M.R. Meireles, P.E. Almeida, M.G. Simoes, A comprehensive review for industrial applicability of artificial neural networks, IEEE Trans. Ind. Electron. 50 (2003) 585-601.

[20] P. Nomikos, J.F. MacGregor, Multi-way partial least squares in monitoring batch processes, Chemom. Intell. Lab. Syst. 30 (1995) 97-108.

[21] H. Noura, D. Sauter, F. Hamelin, D. Theilliol, Fault tolerant control in dynamic systems: application to a winding machine, IEEE Control Mag. 20 (2000) 33-49.

[22] R.J. Patton, Fault tolerant control: the 1997 situation (survey), in: Proceedings of IFAC SAFEPROCESS'97, Hull, 1997. pp. 1033-1055.

[23] P.P. Polycarpou, A.J. Helmicki, Automated fault detection and accommodation: a learning systems approach, IEEE Trans. Syst. Man Cyber. 25 (1995) 1447-1458.

[24] J. Prakash, S.C. Patwardhan, S.A. Narasimhan, Supervisory approach to fault tolerant control of linear multivariable, Syst. Ind. Eng. Chem. Res. 41 (2002) 2270-2281.

[25] T.N. Pranatyasto, S.J. Qin, Sensor validation and process fault diagnosis for FCC units under MPC feedback, Control Eng. Practice 9 (2001) 877-888.

[26] S.J. Qin, Partial least squares regression for recursive system identification, in: Proceedings of the 32th Conference on Decision and Control, San Antonio, 1993, pp. 2617-2622.

[27] S. Qin, Recursive PLS algorithms for adaptive data modeling, Comput. Chem. Eng. 22 (1998) 503514.

[28] S.J. Qin, T.J. McAvoy, Nonlinear PLS modelling using neural networks, Comput. Chem. Eng. 16 (1992) 379-391.

[29] H.E. Rausch, Autonomous control reconfiguration, IEEE Control Syst. Mag. 15 (1995) 37-49.

[30] M. Sourander, T. Liikala, K. Koivisto, FTC strategies in model predictive control of a dearomatisation process, in: H.Y. Zhang (Ed.), Proceedings of the Sixth IFAC Symposium on Fault Detection, Supervision and Safety for Technical Processes, Beijing, 2006, pp. 325-330.

[31] M. Sourander, T. Liikala, M. Vermasvuori, S.-L. Jamsa-Jounela, Offline testing of the FTC-strategy for dearomatisation process of the Naantali refinery, in: Proceedings of the 14th Nordic Process Control Workshop, Espoo, 2007, pp. 120-125.

[32] P. van Overschee, B. de Moor, A unifying theorem for three subspace system identification algorithms, Automatica 31 (1995) 1853-1864.

[33] P. van Overschee, B. de Moor, N4SID: subspace algorithms for the identification of combined deterministic-stochastic systems, Automatica 30 (1996) 75-93.

[34] M. Verhaegen, Identification of the deterministic part of MIMO state space models given in innovations form from input-output data, Automatica 30 (1994) 61-74.

[35] B. Walczak, D.L. Massart, Application of radial basis functions partial least squares to nonlinear pattern recognition problems: diagnosis of process faults, Anal. Chim. Acta 331 (1996) 187-193.

[36] J. Wang, J.S. Qin, A new subspace identification approach based on principal component analysis, $J$. Process Control 12 (2002) 219-224. 
[37] X. Wang, U. Kruger, B. Lennox, Recursive partial least squares algorithms for monitoring complex industrial processes, Control Eng. Practice 11 (2003) 613-632.

[38] S. Wold, H. Martens, H. Wold, The multivariate calibration method in chemistry solved by the PLS method, in: Proceedings of the Conference Matrix Pencils Lecture Notes in Mathematics, 1983, pp. 286-293.

[39] S. Wold, N. Kettaneh-Wold, B. Skagerberg, Nonlinear PLS modeling, Chemom. Intell. Lab. Syst. 7 (1989) 53-65.

[40] S. Wold, Nonlinear partial least squares modelling II spline inner relation, Chemom. Intell. Lab. Syst. 14 (1992) 71-84. 